\title{
Relation between functional magnetic resonance imaging (fMRI) and single neuron, local field potential (LFP) and electrocorticography (ECoG) activity in human cortex
}

\section{George A. Ojemann ${ }^{1 *}$, Nick F. Ramsey ${ }^{2}$ and Jeffrey Ojemann ${ }^{1,3}$}

${ }^{1}$ Neurological Surgery, University of Washington, Seattle, WA, USA

${ }^{2}$ Neurology and Neurosurgery, University Medical Center, Utrecht, Netherlands

${ }^{3}$ Seattle Children's Hospital, Seattle, WA, USA

\section{Edited by:}

Claire Stevenson, Aalto University,

Finland

\section{Reviewed by:}

Gareth Barnes, University College London, UK

Shanqing Cai, Boston University, USA

*Correspondence:

George A. Ojemann, Neurological Surgery, University of Washington, Box 356470, Seattle, WA 98195, USA.

e-mail:gojemann@

u.washington.edu
The relation between changes in the blood oxygen dependent metabolic changes imaged by functional magnetic resonance imaging (fMRI) and neural events directly recorded from human cortex from single neurons, local field potentials (LFPs) and electrocorticogram (ECOG) is critically reviewed, based on the published literature including findings from the authors' laboratories. All these data are from special populations, usually patients with medically refractory epilepsy, as this provides the major opportunity for direct cortical neuronal recording in humans. For LFP and ECoG changes are often sought in different frequency bands, for single neurons in frequency of action potentials. Most fMRI studies address issues of functional localization. The relation of those findings to localized changes in neuronal recordings in humans has been established in several ways. Only a few studies have directly compared changes in activity from the same sites in the same individual, using the same behavioral measure. More often the comparison has been between $\mathrm{fMRI}$ and electrophysiologic changes in populations recorded from the same functional anatomic system as defined by lesion effects; in a few studies those systems have been defined by fMRI changes such as the "default" network. The fMRI-electrophysiologic relationships have been evaluated empirically by colocalization of significant changes, and by quantitative analyses, often multiple linear regression. There is some evidence that the fMRI-electrophysiology relationships differ in different cortical areas, particularly primary motor and sensory cortices compared to association cortex, but also within areas of association cortex. Although crucial for interpretation of $\mathrm{fMRI}$ changes as reflecting neural activity in human cortex, controversy remains as to these relationships. Supported by: Dutch Technology Foundation and University of Utrecht Grant UGT7685, ERC-Advanced grant 320708 (NR) and NIH grant NS065186 (JO)

Keywords: fMRI BOLD, electrocorticogram, single neurons, local field potentials, human cortex
Functional magnetic resonance imaging (fMRI) with the blood oxygen dependent (BOLD) signal has become a major tool to investigate human brain function. The goal of many of these studies is to establish the location and nature of the neuronal events that generate human cognitive processes. However, the BOLD signal that is imaged does not measure neuronal events, but rather hemodynamics. In many of those studies there is an implicit assumption that this signal has an invariant relation to activity of neurons, indeed reflecting their firing rate, an assumption often subsumed in titles of these papers such as "Neural mechanisms ...". In the first decade of fMRI studies this implicit assumption was little challenged. However, a decade ago, Heeger and Ress (2002) raised it, asserting that establishing the relationship between neuronal activity and the fMRI signal "has emerged as one of the most important areas in neuroscience." The resolution of it was considered essential to interpreting fMRI findings.
It is well-established that multiple hemodynamic processes contribute to the BOLD response, in addition to neurophysiological events and several sources of artifacts. Hemodynamic processes include oxygenation level (notably levels of deoxyhemoglobin), blood volume, and blood flow (Kim and Ogawa, 2012), all of which respond to changes in neuronal metabolic demand (Attwell et al., 2010). The relationship between BOLD response parameters such as onset time, amplitude, duration, and post-stimulus undershoot on the one hand, and neuronal activity expressed in terms of firing rate, bandwidthlimited local field potentials (LFPs) and evoked potentials on the other, is still a matter of intense research (Logothetis, 2008). Much of the research on "neurovascular coupling" has been conducted with animals (Logothetis, 2008), but recent opportunities with neurosurgical patients have led to studies comparing BOLD and neuronal activity, to elucidate their relationship. 
To date, simultaneous comparison between the two modalities has only been possible in animals (Logothetis et al., 2001; Niessing et al., 2005). The simultaneous imaging and neuronal recording in visual cortex of anesthetized monkey (Logothetis et al., 2001) is of particular interest. That study indicated that the BOLD signal was most closely related to changes in LFPs, usually considered to reflect the afferent inputs to neurons, and less closely to the firing rate of action potentials which reflects the output of neurons. Whether these findings can be extrapolated to human brain is not clear, and studies with human subjects are needed to further our understanding of the exact nature of neurovascular coupling. Here we review a subset of human studies that have addressed this issue. One of the major goals of fMRI studies is identifying the specific brain region(s) associated with a cognitive process. Neuronal activity that is to be compared to this should be collected with comparable spatial resolution (Hermes et al., 2012). For this reason, we have excluded studies where the standard scalp electroencephalogram (EEG) was the measures of neuronal activity, as the EEG has relatively poor spatial resolution, due to the smoothing properties of scalp and skull. What we have included are those human studies that have recorded neuronal activity with a spatial resolution comparable to fMRI, intracranial recordings of electrocorticogram (ECoG) from subdural or intraparenchymal electrodes and LFPs and single neuron activity from microelectrodes.

These intracranial recordings are performed in a clinical setting. This imposes additional limitations. Perhaps the most serious is the nature of the patient population in whom these procedures are indicated. Essentially all these studies have been done on patients with intractable epilepsy. Whether findings in this population can be generalized to other populations is unknown, even though most studies avoid recording from cortex that has been related to the patient's epilepsy by the presence of interictal discharges, structural lesions or evidence of involvement in seizure onset. However, Bettus et al. (2011) compared BOLD connectivity measures in epileptic and non-epileptic cortex in these patients and found differences including inferring effects of epileptic cortex on the "normal" non-epileptic areas. They suggested that there were widespread alterations in neurovascular coupling in these patients. The presence of widespread hypometabolism in lateral temporal cortex well away from the epileptogenic zone was previously shown with fluorodeyoxyglucose positron emission scans (Engel et al., 1982). A second limitation of the human intracranial studies is that the extent of brain sampled in the recordings is restricted to those clinically relevant for that patient. In practice this often means that ECoG recording through subdural grids or multiple depth electrodes (stereoencephalography) cover wide areas of usually only one hemisphere, while LFP and single neuron recording comes from a much more restricted site, though sometimes in both hemispheres such as both medial temporal lobes including both hippocampi.

At present it is not technically possible or safe to perform simultaneous $\mathrm{fMRI}$ and intracranial recording in humans. There is one study (Ritter et al., 2008) that attempted to overcome this by inferring mass synchronized multiunit neuronal activity from very high frequency $(600 \mathrm{~Hz})$ scalp EEG, which can be recorded during fMRI, a technique which is also applicable to "normal" subjects, but required averaging a remarkably large number of task trials (over 4000), limiting its application. Moreover, as mentioned earlier, direct comparison requires measurement at the same resolution, a requirement not met by EEG. Finally, while there are a relatively large number of reports on ECoG-fMRI relationships from multiple centers, with some replication of findings, at present microelectrode-fMRI relationships have been reported by only two centers, the University of Washington (Ojemann et al., 2010) and University of California Los Angeles (Mukamel et al., 2005; Nir et al., 2007, 2008; Ekstrom et al., 2009). Each used different techniques and sampled different brain regions, with the only replication within the same center.

Apart from the complex mechanisms underlying the BOLD response, the electrophysiological measures are also far from straightforward. Single neuron activity and LFPs from intracortical electrodes measure from very small populations that do not necessarily represent activity of the larger population that shares a particular (sub)function. Hence a correlation with a much larger fMRI voxel is unlikely to yield a strong correlation (Logothetis, 2008). On the other hand an ECoG electrode collects signal from approximately half a million neurons but little is known of what type of neurons (presumably predominantly the large pyramidal cells). With the currently preferred spectral analyses it is becoming clear that many fluctuations are superimposed within neuronal populations, and that they appear to be driven by multiple slow oscillations (0-roughly $30 \mathrm{~Hz}$ ) which may originate from deeper brain structures or from the cortical surface (Buzsáki and Wang, 2012). However, the meaning of the various frequencies and fluctuations is not clear yet, and it is even unclear whether oscillations are at play in higher frequency ranges (Engel et al., 2001) or whether the faster fluctuations merely reflect increased local communication (expressed as broadband gamma power changes) (Miller et al., 2009) (see also Buzsáki and Wang, 2012).

Additionally, comparison of BOLD with electrocortical recordings even in the same subjects is not always straightforward. For one, there is no adequate method for identifying the exact location of surface electrodes, for matching BOLD signal and neural activity. Due to a brainshift following leakage of CSF the cortical surface deforms, causing inaccuracies in matching CT images with electrode positions to presurgical MRI (Hermes et al., 2010). Electrodes can further shift during closing of the dura, making implant photographs inaccurate. One approach would be to smooth the fMRI images and/or interpolate between electrodes, but this does not solve a fundamental issue, namely that electrodes measure only from parenchyma immediately underneath the contact surface (i.e., $2.3 \mathrm{~mm}$ for ECoG). Activity can be quite different from tissue immediately adjacent to an electrode (Freeman et al., 2000; Slutzky et al., 2010), hence a mismatch of a few mm would already impact on the comparison of signals. Second, BOLD changes are observed not only near capillaries but also in draining veins (Roberts et al., 2007) and often in upstream vessels due to inflow effects (Gao and Liu, 2012). Unless special measures are taken to minimize those artifacts (Neggers et al., 2008), BOLD foci will be shifted 
some distance along the draining vessels. Thirdly, the ECoG signals are strongly affected by the method of signal referencing. Most groups use common averaging, but this can cause removal of relevant global signal fluctuations. Alternatively one may use electrodes that face the skull, or are positioned on top of another grid. These different methods yield different results. Despite all these concerns, comparing BOLD to same-resolution neural signals is likely to shed some light on neurovascular coupling.

The technique and findings of the studies reviewed here are presented in Tables 1-3. In those tables, the term LFP refers only to those recorded through microelectrodes, with those recorded from the more widely spaced subdural or depth electrodes referred to as ECoG, as the former are thought to represent activity within 250 microns of the electrode (Katzner et al., 2009), while the latter average over an area of $2.3 \mathrm{~mm}$ square or greater for the type of electrode arrays used in the studies reported here. This ECoG spatial sensitivity is still much better than scalp EEG. Most fMRI studies have sought changes occurring with specific tasks. Those studies are in Tables 1-2. They are further subdivided into those task based change studies where the fMRI and neural activity measures were obtained in the same subject (Table 1) from those where the fMRI and neural activity measures were explicitly compared, but derived from different subjects (Table 2). In all intracranial studies, although the same or very similar tasks are used in all subjects, the technical limitation that requires obtaining the fMRI and neural activity measures at different times, separated by days or longer, already introduces a substantial element of variability. When analyzed in the standard way, group fMRI findings have high reproducibility between repeat fMRIs but individual subject findings do not (Raemaekers et al., 2007). In that study individual subject reproducibility was thought to be related to differences in global signal to noise ratios. A recent report suggests that individual subject reproducibility can be substantially increased by analyzing the scans as relative activation maps normalized as a percentage of local excitation (Voyvodic, 2012). Alternative methods also reduce, but not eliminate, test-retest variability (Bennett and Miller, 2010; Birn, 2012). Obtaining fMRI and electrophysiologic data in different subjects likely introduces even more variability, particularly since electrical stimulation mapping of cortical sites essential for several human cognitive processes in this same patient population, including several language and recent verbal memory measures, has demonstrated substantial individual variability in their location (Ojemann and Dodrill, 1985; Ojemann, 1989; Ojemann et al., 1989). In recent years there has been much interest in the large scale networks identified by correlated spontaneous rest activity in fMRI. Table 3 presents studies of the neural activity correlates of these networks, again divided into those studies where the network investigated was identified on fMRI in the same subjects, and the one where the fMRI data was from different subjects than the neural activity.

The studies of Tables $\mathbf{1}$ and $\mathbf{2}$ investigating task based changes utilize many different behavioral paradigms. Most of them compare neural activity and the BOLD signal during a defined behavioral state to that with a "control" condition, but several look for the similarity of changes with manipulation of one parameter within a behavioral condition [e.g., varying stimulus duration (Huettel et al., 2004) or load in a memory task (Meltzer et al., 2008) or between two anatomic areas in or out of a population visual receptive field (Harvey et al., 2013), or epileptic or non-epileptic cortex (Bettus et al., 2011)]. Moreover, the techniques used to establish BOLD-neural activity relationships fall into several categories. One group of studies examined anatomic colocalization of changes above a statistical threshold. That colocalization was established phenomenologically by visually matching (Puce et al., 1995; Huettel et al., 2004; Meltzer et al., 2008) or by a statistical comparison (Puce et al., 1997; Lachaux et al., 2007; McDonald et al., 2010; Ojemann et al., 2010). These studies address the practical question of what electrophysiologic change is likely to be present when a significant fMRI change is present, but neither analysis establishes a quantitative relation between BOLD and the neural activity. The remaining studies examine correlations between the magnitudes of the BOLD and neural signals, providing a quantitative relationship. In several studies, the neural signal was convoluted with a hemodynamic response function prior to correlation with the BOLD signal (Mukamel et al., 2005; Nir et al., 2007; Privman et al., 2007). Most correlation studies have evaluated the BOLD neural activity relationship statistically within a general multiple linear regression model. Given that different tasks are expected to elicit different localization of BOLD changes, the BOLD-electrophysiology coupling for different tasks might also differ. Few studies provide direct evidence on this. Conner et al. (2011) found no difference in ECoG correlative coupling for visually cued object naming or verb generation and Engell et al. (2012) found similar correlative coupling for different visual checkerboard stimulus durations, regardless of the presence or absence of a modulating effect of that parameter on BOLD and ECoG.

What can we conclude from these studies? Despite the differing sites sampled, tasks used and techniques for establishing BOLD-neural activity relationships, there are several. Most studies that have examined task based fMRI-neural activity relations in primary cortical areas, motor, somatosensory, auditory and visual, have reported a relation between increased BOLD signal and increased ECoG or LFP power in the "gamma" $30-130 \mathrm{~Hz}$ range (Tables 1 and 2 ). Single neuron firing has a similar relationship but only when there is correlated firing of nearby neurons. Then, single neuron firing has a quantitative linear relationship with BOLD activation. This is similar to the BOLD-neural activity relationship in monkey visual cortex recorded by Logothetis et al. (2001). However, in the Logothetis et al. study the relationship was to the lower range of "gamma" frequencies. One human study also found the occipital lobe relationship to this lower "gamma" range (Privman et al., 2007). Another human study that tried to directly replicate the Logothetis findings reported a similar positive relationship between the sustained evoked potentials and BOLD increases in peri-calcarine cortex but no consistent BOLD relationship to ECoG power, and no consistent relationship between BOLD and EPs as well as ECoG power in adjacent fusiform gyrus (Huettel et al., 2004). A recent reanalysis of the same data for non-phase-locked changes in high 


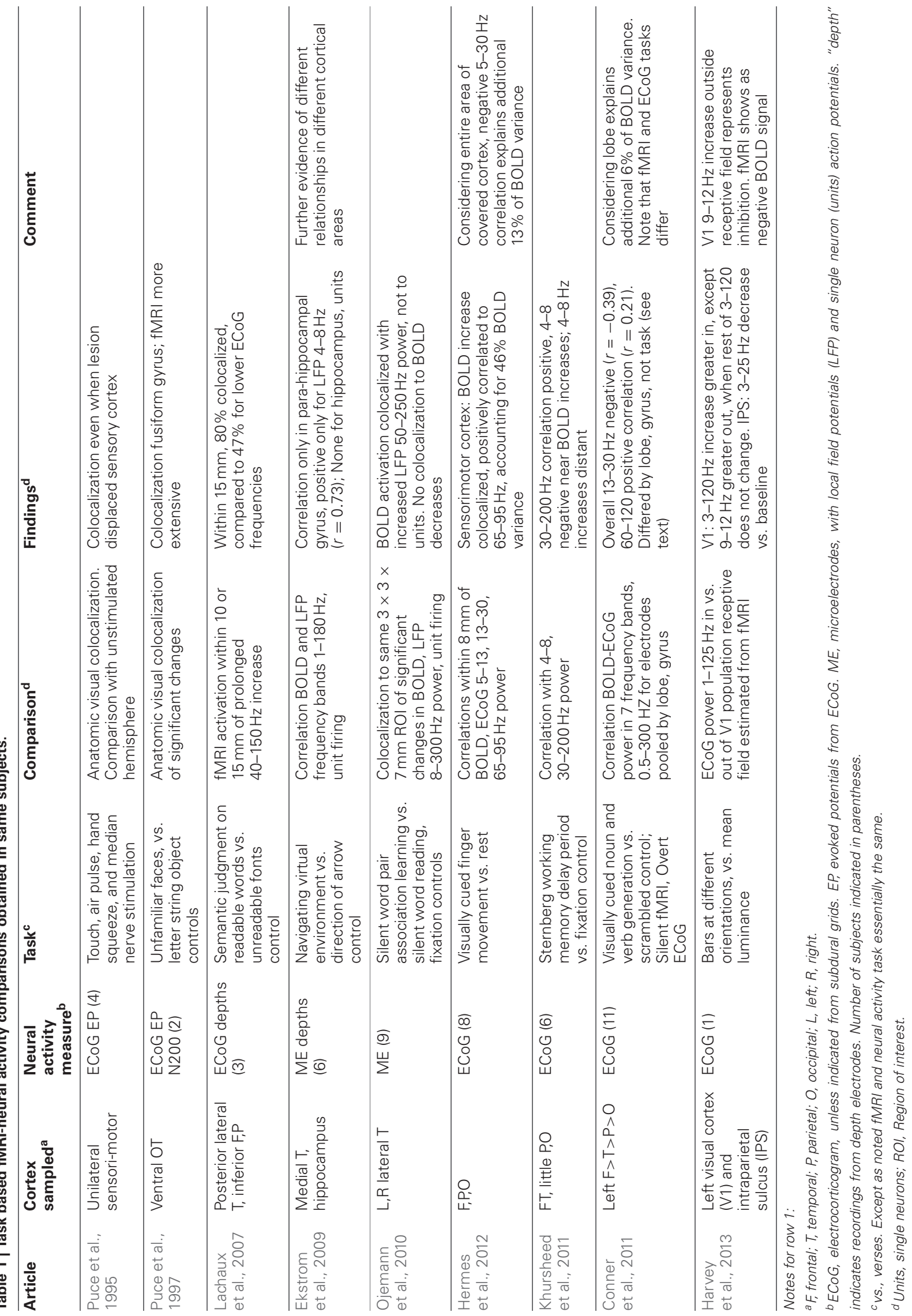




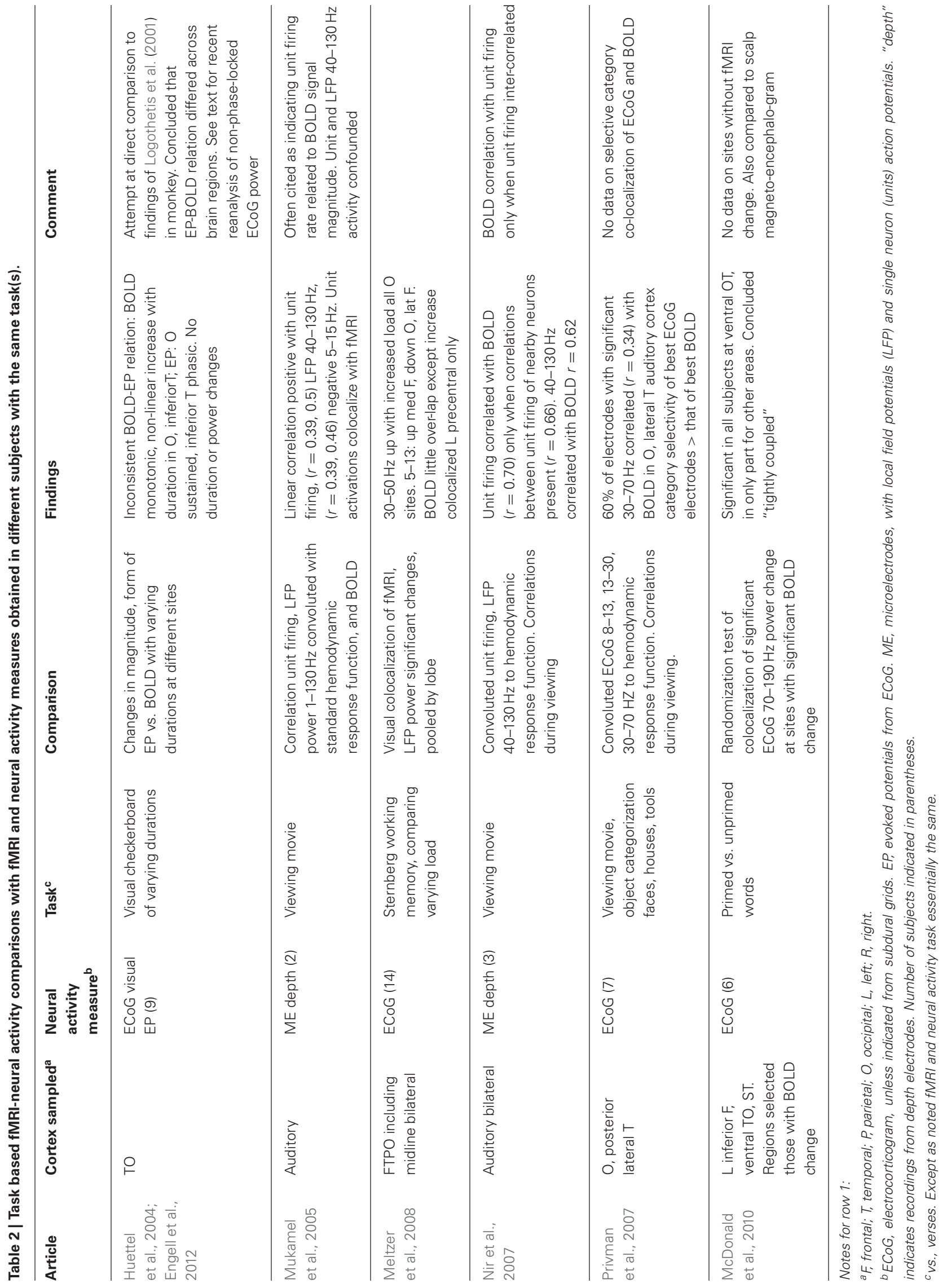




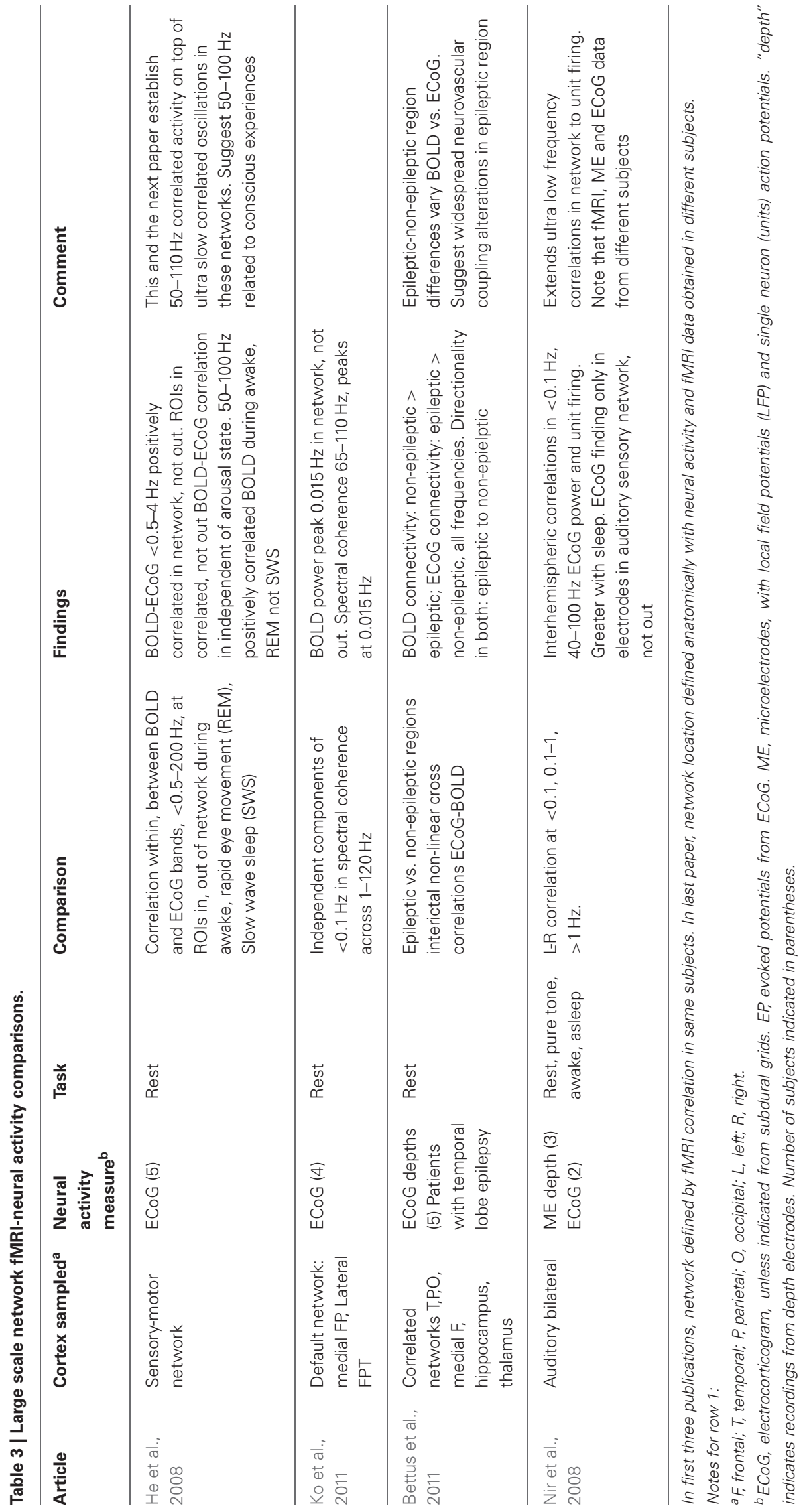


frequency power found a good correspondence between broadband gamma $(30-100 \mathrm{~Hz})$ and BOLD in both areas (Engell et al., 2012).

Increases in power in lower frequencies of ECoG-LFP also have a correlation with BOLD in these primary cortices but this relationship is negative; that increased power may be associated with either a negative BOLD signal (Harvey et al., 2013) or a smaller but still significant increase in the BOLD signal (Hermes et al., 2012). This relationship is less robust and somewhat independent of the relationship to "gamma" increases. The exact "low" frequency range has not been established, 9-12 Hz (Harvey et al., 2013), 5-15 Hz (Mukamel et al., 2005), $5-30 \mathrm{~Hz}$ (Hermes et al., 2012). Since there is increasing evidence that low frequencies are associated with suppression of cortical activity (e.g., Haegens et al., 2012; Miller et al., 2012; Harvey et al., 2013), tending to anticorrelate in amplitude with broadband gamma power (e.g., Hermes et al., 2012; Miller et al., 2012), it may be that there are no specific frequencies that drive the BOLD response.

The situation in association cortex is even less clear, but there too, despite the diversity of sites and tasks, most studies recording from neocortex have identified some positive correlation or colocalization with ECoG or LFP "gamma" frequencies, from $30-250 \mathrm{~Hz}$. A few have also reported a negative correlation with increased power at lower frequencies, again in different ranges, 13-30 Hz (Conner et al., 2011) or 4-8 Hz (Khursheed et al., 2011). A relationship of BOLD to single neuron firing rates has not been established for association cortex. This may be due to the low firing rates of neurons there and the infrequent occurrence of correlated firing between nearby neurons (Ojemann et al., 2002).

Several studies suggest that the BOLD-neural activity relationships differ in different regions of cortex, even for the same task. Huettel et al. (2004) reported differences in the relationship between BOLD and ECoG evoked responses in peri-calcarine or fusiform cortex, though when analyzed by non-phase-locked ECoG power, these differences were not evident (Engell et al., 2012). In recordings from a large number of electrodes in wide areas of the left hemisphere during a language task, Conner et al. (2011) compared the BOLD-ECoG coupling when recordings were pooled by lobe to that of all areas, a positive correlation at $60-120 \mathrm{~Hz}$ and a negative one at $13-30 \mathrm{~Hz}$. Significant differences from that average pattern of coupling were found for all lobes. They also compared the differences in BOLD-ECoG coupling for electrodes pooled by the relation to sites identified as critical for language based on electrical stimulation mapping (Ojemann et al., 1989) to those that were not. Critical language sites in superior temporal gyrus had greater coupling for $120-240 \mathrm{~Hz}$, those in middle temporal gyrus less for $30-120 \mathrm{~Hz}$, and in posterior inferior frontal gyrus (Broca's area), more for $30-240 \mathrm{~Hz}$. fMRI activation has not shown sufficient specificity to these crucial language sites in individual patients to be a substitute for electrical stimulation mapping (Giussani et al., 2010 for review). In addition to the differences between neocortical areas discussed above, Ekstrom et al. (2009) found no significant coupling between BOLD and LFP power or single neuron firing in hippocampus, and the only relationship in the allocortex of the parahippocampal gyrus a positive correlation to $4-8 \mathrm{~Hz}$ LFP power. In those structures there was little relation between LFP "gamma" (or "theta") power and single neuron firing (Ekstrom et al., 2007).

How much such regional differences reflect different degrees of engagement of different areas of cortex by a particular task, or inherent differences in the neurovascular coupling of different areas, or differences in their neuronal populations in their LFP power responsiveness is unknown (Ekstrom, 2010). Some insight into this issue comes from a study of the ability of LFPs in different frequency ranges to predict within $1 \mathrm{~ms}$ the timing of firing of individual neurons recorded through the same microelectrode, in recordings from human lateral temporal cortex (Zanos et al., 2012). Many of the neurons in which such a relationship could be established divided into two separate populations, those with timing of discharges predicted by $8-14 \mathrm{~Hz}$ LFP, and those by $80-150 \mathrm{~Hz}$. Many of the recordings analyzed in that study were performed during the word association learning paradigm used in the Ojemann et al. (2010) study of BOLDLFP relationships. Neurons with firing predicted by $80-150 \mathrm{~Hz}$ significantly increased activity with the learning paradigm, those with $8-14 \mathrm{~Hz}$, to the word reading control. Experimental animal modeling of LFPs analyzed this way indicates that the $80-150 \mathrm{~Hz}$ activity represents input from neighboring neural circuits, while low frequencies originate from distant neuronal populations. In the Ojemann et al. (2010) study BOLD increased signal during the learning paradigm, compared to the control, colocalized with $50-250 \mathrm{~Hz}$ LFP power increases, suggesting that the BOLD signal reflects local synaptic input.

The large scale networks identified by rest activity in the fMRI that correlates across wide areas colocalize with low to ultra low frequency ECoG oscillations, less than $0.5-4 \mathrm{~Hz}$ in one study, less than 0.1 in two others, with a peak at $0.015 \mathrm{~Hz}$ in one (Table 3). This relationship has been identified in several large scale networks, including sensory-motor and the "default" network. Riding on these very slow oscillations are $40-110 \mathrm{~Hz}$ oscillations, which in the study of Ko et al. (2011) were most prominent at $0.015 \mathrm{~Hz}$ frequency of the slow oscillations. That single neuron firing rates correlate with the very slow oscillations is suggested in the one study where this was examined, where however, single neuron, ECoG and fMRI data were collected from different patients.

The above findings establish broadband "gamma" $(30-250 \mathrm{~Hz})$ ECoG-LFP power as a major feature of the neural activity coupled to the task based BOLD metabolic signal in humans in neocortex, independent of the specific task, thus providing a partial answer to Heeger and Ress challenge. However, it is also clear that the coupling may not be so simple as the invariant linear relation to neuron firing rates assumed in the early days of fMRI. Many aspects remain to be established, among them the role of decreases in power of lower ECoGLFP frequencies, the extent and causes of the spatial variability in the coupling to the ECoG-LFP, the conditions under which the BOLD signal does reflect single neuron firing, and if so the nature of the relation, and the role of the ultra slow frequency oscillations of the large scale networks in modulating neural activity. 


\section{REFERENCES}

Attwell, D., Buchan, A. M., Charpak, S., Lauritzen, M., Macvicar, B. A., and Newman, E. A. (2010). Glial and neuronal control of brain blood flow. Nature 468, 232-243.

Bennett, C. M., and Miller, M. B. (2010). How reliable are the results from functional magnetic resonance imaging? Ann. N. Y. Acad. Sci. 1191, 33-55.

Bettus, G., Ranjeva, J.-P., Wendling, F., Benar, C., Confort-Gouny, S., Regis, J., et al. (2011). Interictal functional connectivity of human epileptic networks assessed by intracerebral EEG and BOLD signal fluctuations. PLoS ONE 6:e20071. doi: 10.1371/ journal.pone.0020071

Birn, R. M. (2012). The role of physiological noise in resting-state functional connectivity. Neuroimage 62, 864-870.

Buzsáki, G., and Wang, X. J. (2012). Mechanisms of gamma oscillations. Annu. Rev. Neurosci. 35, 203-225.

Conner, C. R., Ellmore, T. M., Peters, T. A., DiSano, M. A., and Tandon, N. (2011). Variability of the relationship between electrophysiology and BOLD-fMRI across cortical regions in humans. J. Neurosci. 31, 12855-12865.

Ekstrom, A. (2010). How and why the fMRI BOLD signal relates to underlying neural activity: the danger in dissociation. Brain Res. Rev. 62, 233-244.

Ekstrom, A., Suthana, N., Millett, D., Fried, I., and Bookheimer, S. (2009). Correlation between BOLD fMRI and theta-band local field potentials in the human hippocampal area. J. Neurophysiol. 101, 2668-2678.

Ekstrom, A., Viskontas, I., Kahana, M., Jacobs, J., Upchurch, K., Bookheimer, S., et al. (2007). Contrasting roles of neural firing rate and local field potentials in human memory. Hippocampus 17, 606-617.

Engel, A. K., Fries, P., and Singer, W. (2001). Dynamic predictions: oscillations and synchrony in top-down processing. Nat. Rev. Neurosci. 2, 704-716.

Engel, J. Jr., Kuhl, D. E., Phelps, M. E., and Mazziota, J. C. (1982). Interictal cerebral glucose metabolism in partial epilepsy and its relation to EEG change. Ann. Neurol. 12, 510-517.

Engell, A. D., Huettel, S., and McCarthy, G. (2012). The fMRI BOLD signal tracks electrophysiological spectral perturbations, not event-related potentials. Neuroimage 59, 2600-2606.
Freeman, W. J., Rogers, L. J., Holmes, M. D., and Silbergeld, D. L. (2000). Spatial spectral analysis of human electrocorticograms including the alpha and gamma bands. J. Neurosci. Methods 95, 111-121.

Gao, J. H., and Liu, H. L. (2012). Inflow effects on functional MRI. Neuroimage 62, 1035-1039.

Giussani, C., Roux, F.-E., Ojemann, J., Sganzerla, E. P., Pirillo, D., and Papagno, C. (2010). Is preoperative functional magnetic resonance imaging reliable for language areas mapping in brain tumor surgery? Review of language functional magnetic imaging and direct cortical stimulation studies. Neurosurgery 66, 113-120.

Haegens, S., Luther, L., and Jensen, O. (2012). Somatosensory anticipatory alpha activity increases to suppress distracting input. J. Cogn. Neurosci. 24, 677-685.

Harvey, B. M., Vansteensel, M. J., Ferrier, C. H., Petridou, N., Zuiderbaan, W., Aarnoutse, E. J., et al. (2013). Frequency specific spatial interactions in human electrocorticography: V1 alpha oscillations reflect surround suppression. Neuroimage 65 424-432.

He, B. J., Snyder, A. Z., Zempel, J. M., Smyth, M. D., and Raichle, M. E. (2008). Electrophysiological correlates of the brain's intrinsic large-scale functional architecture. Proc. Natl. Acad. Sci. U.S.A. 105, 16039-16044.

Heeger, D. J., and Ress, D. (2002). What does fMRI tell us about neuronal activity? Nat. Rev. Neurosci. 3, 142-151.

Hermes, D., Miller, K. J., Noordmans, H. J., Vansteensel, M. J., and Ramsey, N. F. (2010). Automated electrocorticographic electrode localization on individually rendered brain surfaces. J. Neurosci. Methods 185, 293-298.

Hermes, D., Miller, K. J., Vansteensel, M. J., Aarnoutse, E. J., Leijten, F. S. S., and Ramsey, N. F. (2012). Neurophysiologic correlates of fMRI in human motor cortex. Hum. Brain Mapp. 33, 1689-1699.

Huettel, S. A., McKeown, M. J., Song, A. W., Hart, S., Spencer, D. D., Allison, T., et al. (2004). Linking hemodynamic and electrophysiological measures of brain activity: evidence from functional MRI and intracranial field potentials. Cereb. Cortex 14, 165-173.

Katzner, S., Nauhaus, I., Benucci, A., Bonin, V., Ringach, D. L., and Carandini, M. (2009). Local origin of field potentials in visual cortex. Neuron 61, 35-41.

Khursheed, F., Tandon, N., Tertel, K., Pieters, T. A., Disano, M. A., and Ellmore, T. M. (2011). Frequencyspecific electrocorticographic correlates of working memory delay period fMRI activity. Neuroimage 56, 1773-1782.

Kim, S. G., and Ogawa, S. (2012). Biophysical and physiological origins of blood oxygenation level-dependent fMRI signals. J. Cereb. Blood Flow Metab. 32, 1188-1206.

Ko, A., Darvas, F., Poliakov, A., Ojemann, J., and Sorenson, L. B. (2011). Quasi-periodic fluctuations in default mode network electrophysiology. J. Neurosci. 31, 11728-11732.

Lachaux, J.-P., Fonlupt, P., Kahane, P., Minotti, L., Hoffman, D., Bertrand, O., et al. (2007). Relationship between task-related gamma oscillations and BOLD signal: new insights from combined fMRI and intracranial EEG. Hum. Brain Mapp. 28, 1368-1375.

Logothetis, N. K. (2008). What we can do and what we cannot do with fMRI. Nature 453, 869-878.

Logothetis, N. K., Pauls, J., Augath, M., Trinath, T., and Oeltermann, A. (2001). Neurophysiological investigation of the basis of the fMRI signal. Nature 412, 150-157.

McDonald, C. R., Thesen, T., Carlson, C., Blumberg, M., Girard, H. M., Trongnetrpunya, A., et al. (2010). Multimodal imaging of repetition priming: using fMRI, MEG and intracranial EEG to reveal spatiotemporal profiles of word processing. Neuroimage 53, 707-717.

Meltzer, J. A., Zaveri, H. P., Goncharova, I. I., Distasio, M. M., Papademetris, X., Spencer, S. S., et al. (2008). Effects of working memory load on oscillatory power in human intracranial EEG. Cereb. Cortex 18, 1843-1855.

Miller, K. J., Hermes, D., Honey, C. J., Hebb, A. O., Ramsey, N. F., Knight, R. T., et al. (2012). Human motor cortical activity is selectively phaseentrained on underlying rhythms. PLoS Comput. Biol. 8:e1002655. doi: 10.1371/journal.pcbi.1002655

Miller, K. J., Sorensen, L. B., Ojemann, J. G., and den Nijs, M. (2009). Power-law scaling in the brain surface electric potential. PLoS Comput. Biol. 5:e1000609. doi: 10. 1371/journal.pcbi.1000609

Mukamel, R., Gelbard, H., Arieli, A., Hasson, U., Fried, I., and Malach, R.
(2005). Coupling between neuronal firing, field potentials and fMRI in human auditory cortex. Science 309, 951-954.

Neggers, S. F., Hermans, E. J., and Ramsey, N. F. (2008). Enhanced sensitivity with fast three-dimensional blood-oxygenlevel-dependent functional MRI: comparison of SENSE-PRESTO and 2D-EPI at 3 T. NMR Biomed. 21, 663-676.

Niessing, J., Ebisch, B., Schmidt, K. E., Niessing, M., Singer, W., and Galuske, R. A. (2005). Hemodynamic signals correlate tightly with synchronized gamma oscillations. Science 309, 948-951.

Nir, Y., Fisch, L., Mukamel, R., GelbardSagiv, H., Arielli, A., Fried, I., et al. (2007). Coupling between neuronal firing rate, gamma LFP, and BOLD fMRI is related to interneuronal correlations. Curr. Biol. 17, 1275-1285.

Nir, Y., Mukamel, R., Dinstein, I., Privman, E., Harel, M., Fisch, L., et al. (2008). Interhemispheric correlations of slow spontaneous neuronal fluctuations revealed in human sensory cortex. Nat. Neurosci. 11, 1100-1108.

Ojemann, G., Ojemann, J., Lettich, E., and Berger, M. (1989). Cortical language localization in left, dominant hemisphere: an electrical stimulation mapping investigation in 117 patients. J. Neurosurg. 71, 316-326.

Ojemann, G. A. (1989). "Some brain mechanisms for reading," in Brain and Reading, eds C. Von Euler, I. Lundberg, and G. Lennerstrand (Hampshire: MacMillan Press Ltd), 47-59.

Ojemann, G. A., Corina, D. P., Corrigna, N., Schoenfield-McNeill, J., Poliakov, A., Zamora, L., et al. (2010). Neuronal correlates of functional magnetic resonance imaging in human temporal cortex. Brain 133, 46-59.

Ojemann, G. A., and Dodrill, C. B. (1985). Verbal memory deficits after left temporal lobectomy for epilepsy: mechanism and intraoperative prediction. J. Neurosurg. 62, 101-107.

Ojemann, G. A., Schoenfield-McNeill, J., and Corina, D. (2002). Anatomic subdivisions in human temporal cortical neuronal activity related to recent verbal memory. Nat. Neurosci. 5, 64-71.

Privman, E., Nir, Y., Kramer, U., Kipervasser, S., Andelman, F., Neufeld, M. Y., et al. (2007). Enhanced category tuning revealed by intracranial 
electroencephalograms in higherorder human visual areas. J. Neurosci. 27, 6234-6242.

Puce, A., Allison, T., Spencer, S. S., Spencer, D. D., and McCarthy, G. (1997). Comparison of cortical activation evoked by faces measured by intracranial field potentials and functional fMRI; two case studies. Hum. Brain Mapp. 5, 298-305.

Puce, A., Constable, R. T., Luby, M. L., McCarthy, G., Nobre, A. C., Spencer, D. D., et al. (1995). Functional magnetic resonance imaging of sensory and motor cortex: comparison with electrophysiological localization. J. Neurosurg. 83, 262-270.

Raemaekers, M., Vink, M., Zandbelt, B., van Wezel, R. J. A., Kahn, R. S., and Ramsey, N. F. (2007). Test-retest reliability of fMRI activation during prosaccades and antisaccades. Neuroimage 36, 532-542.

Ritter, P., Freyer, F., Curio, G., and Villringer, A. (2008). High-frequency $(600 \mathrm{~Hz})$ population spikes in human EEG delineate thalamic and cortical fMRI activation sites. Neuroimage $42,483-490$.

Roberts, K. C., Tran, T. T., Song, A. W., and Woldorff, M. G. (2007). Component structure of eventrelated fMRI responses in the different neurovascular compartments. Magn. Reson. Imaging 25, 328-334.

Slutzky, M. W., Jordan, L. R., Krieg, T., Chen, M., Mogul, D. J., and Miller, L. E. (2010). Optimal spacing of surface electrode arrays for brain-machine interface applications. J. Neural Eng. 7, 26004.

Voyvodic, J. (2012). Reproducibility of single-subject fMRI language mapping with AMPLE normalization. J. Magn. Reson. Imaging 36, 569-580.

Zanos, S., Zanos, T. P., Marmarelis, V. Z., Ojemann, G. A., and Fetz, E. E. (2012). Relationships between spike-free local field potentials and spike timing in human temporal cortex. J. Neurophysiol. 107, 1808-1821.

Conflict of Interest Statement: The authors declare that the research was conducted in the absence of any commercial or financial relationships that could be construed as a potential conflict of interest.
Received: 07 November 2012; accepted: 29 January 2013; published online: 20 February 2013.

Citation: Ojemann GA, Ramsey NF and Ojemann J (2013) Relation between functional magnetic resonance imaging ( $\mathrm{PMRI}$ ) and single neuron, local field potential (LFP) and electrocorticography (ECoG) activity in human cortex. Front. Hum. Neurosci. 7:34. doi: 10.3389/fnhum.2013.00034

Copyright (c) 2013 Ojemann, Ramsey and Ojemann. This is an open-access article distributed under the terms of the Creative Commons Attribution License, which permits use, distribution and reproduction in other forums, provided the original authors and source are credited and subject to any copyright notices concerning any third-party graphics etc. 